\title{
IMAGE REGISTRATION OF HIGH-RESOLUTION UAV DATA: THE NEW HYPARE ALGORITHM
}

\author{
T. Bahr ${ }^{\text {a, }}$, X. Jin ${ }^{\text {b }}$, R. Lasica ${ }^{\text {b }}$, D. Giessel ${ }^{\text {c }}$ \\ a Exelis Visual Information Solutions GmbH, Gilching, Germany - thomas.bahr@exelisvis.com \\ ${ }^{\mathrm{b}}$ Exelis Visual Information Solutions, Boulder, Colorado, U.S.A - (xiaoying.jin, rebecca.lasica)@exelisvis.com \\ ${ }^{\mathrm{c}}$ Aeryon Labs Inc., Waterloo, Ontario, Canada - dgiessel@aeryon.com
}

KEY WORDS: Image Registration, UAV, Optical Data, Automation, HyPARE, Algorithm

\begin{abstract}
:
Unmanned aerial vehicles play an important role in the present-day civilian and military intelligence. Equipped with a variety of sensors, such as SAR imaging modes, E/O- and IR sensor technology, they are due to their agility suitable for many applications. Hence, the necessity arises to use fusion technologies and to develop them continuously. Here an exact image-to-image registration is essential. It serves as the basis for important image processing operations such as georeferencing, change detection, and data fusion. Therefore we developed the Hybrid Powered Auto-Registration Engine (HyPARE). HyPARE combines all available spatial reference information with a number of image registration approaches to improve the accuracy, performance, and automation of tie point generation and image registration. We demonstrate this approach by the registration of 39 still images from a high-resolution image stream, acquired with a Aeryon Photo3 $\mathrm{S}^{\mathrm{TM}}$ camera on an Aeryon Scout micro-UAV ${ }^{\mathrm{TM}}$.
\end{abstract}

\section{INTRODUCTION}

Image registration involves locating and matching tie points in the two images to be registered. In manual registration, a human operator performs these tasks visually using interactive software. The process is repetitive, laborious, tedious and prone to error. Manual registration becomes prohibitive for the large amount of data, e.g. resulting from image streams acquired by UAVs, and large geographic coverage.

An automated registration solution is highly desired in a production environment. In automated registration, algorithms automatically generate tie points and align the image. However, especially still images from UAV payloads have various characteristics that make automated registration difficult. Location errors may occur in the navigation and during UAV maneuvers. Various image characteristics due to multi-temporal effects, terrain effects, different sensor modalities and different spatial resolution all make automated registration a challenging task. Thus, an automated registration does not always offer the required reliability and accuracy; often manual editing of tie points is needed.

To solve these requirements we developed the Hybrid Powered Auto-Registration Engine (HyPARE). This registration engine generates tie points automatically and accurately. It takes advantage of all the available spatial reference information to achieve the best accuracy and automation during registration. It combines a number of registration approaches to improve the reliability, accuracy, performance and automation of image registration. It is robust in the situations of images obtained from different viewing angles, in different time and seasons, with various terrain relief, and by sensors with different modalities, e.g. SAR and E/O. The algorithm is automated and optimized so that user interaction and editing is minimized or eliminated (Jin, 2012).

\section{THE HYBRID POWERED AUTO-REGISTRATION ENGINE (HYPARE)}

HyPARE combines all available spatial reference information to achieve the best accuracy and automation during registration (see Figure 1). The spatial reference information may come from the following sources (Jin, 2012):

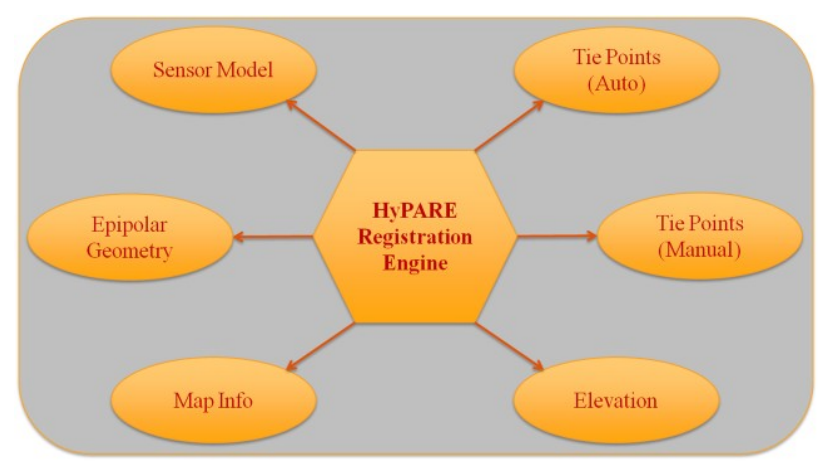

Figure 1. Key components of the Hybrid Powered AutoRegistration Engine (HyPARE)

Standard map information or RPC information of the input images establishes the approximate geometric relationship between a warp image and a base image. It reduces the search space and improves the reliability of automatic tie point generation.

For most applications, the tie point information is automatically generated with no or minimized number of outliers. The operator can manually define a few seed tie points and feed them into automatic tie point generation to improve the overall accuracy.

Geo-location geometric constraints are used to search for and filter tie points. The images should align well in the common ground coordinate space in the orthorectified images or nadir 
view images; therefore, all the tie points are constrained by a global transform between the coordinates in the base image and the warp image.

RPC sensor models and elevation are used for orthorectification on-the-fly during image registration. This geometrically corrects the data to a common ground coordinate space, and achieves better accuracy in automatic tie point finding and filtering. The tie points can be automatically generated even in the image areas with big distortion during to terrain relief, such as mountainous areas.

For images taken at different viewing positions and/or angles, the images of the same scene are related by the epipolar geometry constraint. For a feature point in the first image, the corresponding point in the second image must lie on the epipolar line or curve. Two epipolar geometry models are used in geometric filtering: one is suitable for the imagery with the frame central projection, such as acquired by multispectral UAV payloads, and the other is suitable for the imagery obtained with a pushbroom sensor that has RPC information.

\section{THE IMAGE REGISTRATION WORKFLOW}

For the application of the registration process we embedded the HyPARE algorithm in a wizard guiding through file selection, automatic tie point generation, review of the tie points and preview of the warped image, and export of image registration results and tie points (Jin, 2012).

The workflow starts with the selection of a base image as the reference image and a warp image to be registered with the base image. The base image can have standard map projections or RPC information; it cannot be pixel-based, arbitrary, or a pseudo projection. The warp image does not have restrictions. If a pixel-based image or an image with pseudo projection or arbitrary projection is used as the warp image, one has to select at least three seed tie points to define the approximate relationship between the two images.

Auto tie point generation compares the gray scale values of patches of two images and tries to find joined image locations based on similarity in those gray scale value patterns. Two image matching methods are available: a correlation-based method and a mutual information-based method. The correlation-based method is e.g. applicable for optical UAV image sequences, as demonstrated in the case study (see section 4). The mutual information-based method is optimized for registering images with different modalities. The normalized mutual information between the patch in the base image and the patch in the warp image is computed as the matching score. Mutual information is based on information theory and measures the mutual dependence of the two random variables (Exelis Visual Information Solutions (Ed.), 2012). The false tie points with a low matching score are removed. The outliers are further removed by fitting and filtering by a geometric model. The operator can select the geometric model from a global transform model or two epipolar geometry based models.

If the base image or warp image includes RPC information, we recommend the use of a DEM file. The registration engine performs orthorectification on-the-fly, and generates tie points in the common ground coordinate space. The image is geometrically corrected for automatic tie point generation. Radiometric matching and geometric filtering are performed in the common ground coordinate space to improve the reliability and accuracy of tie point generation.

The automatic tie point generation algorithm is optimized to minimize or eliminate the need of manual tie point editing. However, one can choose to review, add, or delete tie points interactively. Further options within this processing step are changing the image warping and resampling parameters and previewing the image warping result.

At the end, one can export the warped image which is registered with the base image, and the tie points to be used in other applications or systems.

\section{CASE STUDY: REGISTRATION OF STILL IMAGES FROM UAV ACQUSITIONS}

For the present case study we imported 39 still images from a colour image stream acquired with the Photo $3 \mathrm{~S}^{\mathrm{TM}}$ camera by Aeryon Labs Inc., the industry's only integrated 3-axis stabilized high-resolution camera for micro UAVs. It was mounted on an Aeryon Scout micro-UAV ${ }^{\mathrm{TM}}$ (see Figure 2) for a flight over Bloomingdale, Ontario, Canada on Nov. 272012 (Aeryon Labs Inc. (Ed.), 2013). The flight altitude was approx. $50 \mathrm{~m}$; from this resulted a GSD of $1.5 \mathrm{~cm}$.

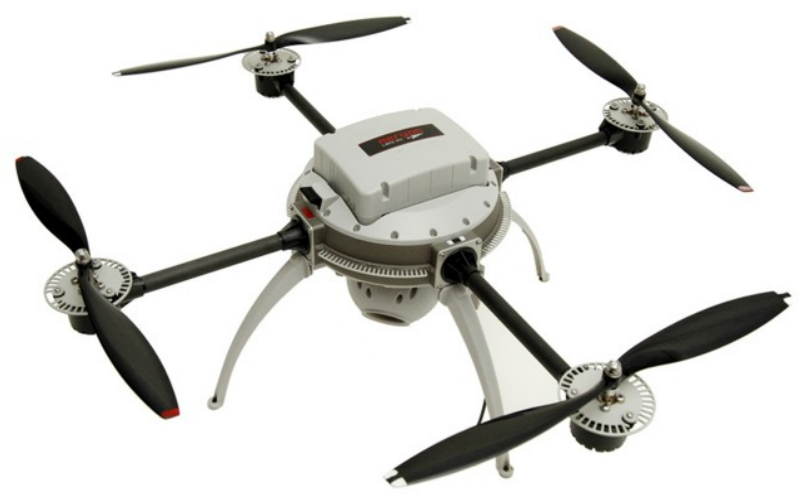

Figure 2. Aeryon Scout micro-UAV ${ }^{\mathrm{TM}}$ with the Photo3S ${ }^{\mathrm{TM}}$ camera mounted. (C) Aeryon Labs Inc. 2012, all rights reserved.

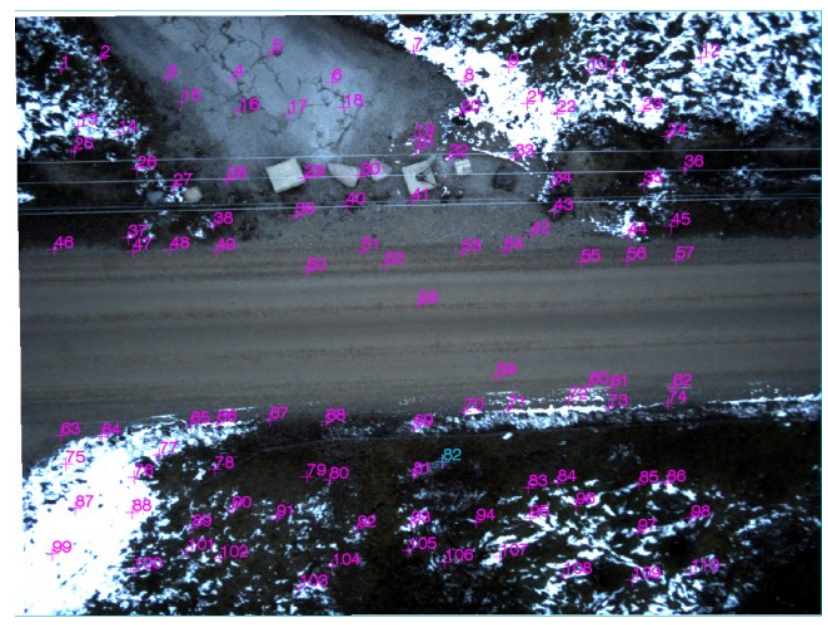

Figure 3. Photo $3 \mathrm{~S}^{\mathrm{TM}}$ colour still image with automatically calculated tie points, Bloomingdale, Ontario, Canada. (C) Aeryon Labs Inc. 2012, all rights reserved. 
Prior to the registration process the images were automatically georeferenced with ENVI on the basis of IDL (Exelis Visual Information Solutions (Ed.), 2012), using the centre coordinates in Geographic Lat/Lon as a geodetic reference.

Figure 3 shows one of the warp images with 110 automatically calculated tie points, well distributed across the overlapping areas. After image registration, the features of the warped image align accurately with the base image (see Figure 4). The effects of variable pitch, roll, and yaw angles were successfully compensated.

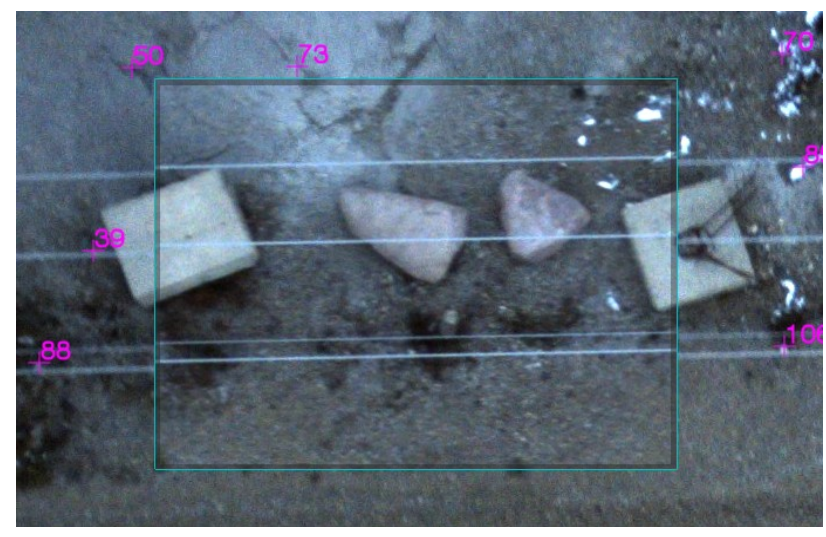

Figure 4. Photo3 $\mathrm{S}^{\mathrm{TM}}$ image registration preview, Bloomingdale, Ontario, Canada. (C) Aeryon Labs Inc. 2012, all rights reserved.

Subsequently, the 39 images were stitched using ENVI's mosaicing functionality (Exelis Visual Information Solutions (Ed.), 2012).

The complete workflow used for this case study - consisting of georeferencing, image registration, and mosaicing - can be created as a non-interactive IDL batch process. This even allows to integrate this automated workflow in other platforms, such as ArcGIS $^{\mathrm{TM}}$.

\section{DISCUSSION}

In this case study we didn't consider the input of a highresolution digital elevation model to compensate effects due to terrain relief. The Aeryon payload is stabilized in all three axes. Thus the still images used are all within better than $0.5^{\circ}$ off nadir. According to Aeryon Labs Inc. (Ed.), 2013, a typical value is $0.1^{\circ}$ or less. Crosswinds, tailwinds, and the ends of flight lines do not affect this perspective, as the quad rotor type UAV (see Figure 2) does not have to (1) "crab" into crosswinds, (2) maintain a minimum airspeed to prevent stalling (the main reason tail winds affect fixed wings), and (3) experience high bank angles, as it can vector its thrust with very minor changes in its orientation. These changes can be easily compensated by the payload gimbal. This ensures that all of the images are very close to nadir and have minimal skew relative to each other.

Another subject of discussion are the off-nadir ray-angles for any portion of the image that is not at the exact centre of the frame. Generally, they result from the low altitude of data collection and relatively wide viewing angle of the camera (certainly wide when compared to satellite imagery). This change in perspective near the edge of the frame relative to its centre would manifest itself in the same way as taking a slightly oblique image. However, the image registration workflow presented here allows to review and delete tie points interactively (see section 2). Thus the stitching process can be the confined on the centre portion of the images. Additionally, the HyPARE algorithm is robust in the situation of images obtained from different viewing angles. Moreover, datasets with a high image overlap, as used in this case study, have more atnadir image centres to crop out and stitch together, so less of the distorted periphery must be used. This ensures that the off-nadir ray-angles are of minor importance within the stitching process.

\section{CONCLUSIONS}

With the Hybrid Powered Auto-Registration Engine (HyPARE) we developed an approach to generate tie points accurately and geometrically align images automatically. HyPARE is robust in situations of images obtained from different viewing angles, in different time and seasons, with various terrain relief, and by sensors with different modalities. Therefore it is suitable for many applications.

Thus we solved an important requirement for today's UAV E/O, IR, and SAR sensors. We demonstrated this successfully by the registration of 39 still images from a high-resolution image stream, acquired with a Aeryon Photo $3 \mathrm{~S}^{\mathrm{TM}}$ camera on an Aeryon Scout micro-UAV ${ }^{\mathrm{TM}}$.

As the field of remote sensing keeps evolving, we are continuing our research and development on new solutions to meet the changing requirements and scope of image registration and its related projects. Future direction is to extend the automatic registration algorithm to imagery with mostly forest and desert areas, images with considerable shadows and clouds, images acquired from highly off-nadir views, etc. Future work will also include to automatically register images to LiDAR point clouds, and to register images to maps such as GIS vector layers (Jin, 2012).

\section{REFERENCES}

Aeryon Labs Inc. (Ed.), 2013. Aeryon small Unmanned Aerial Systems, http://www.aeryon.com/products/, 20.02.2013.

Exelis Visual Information Solutions (Ed.), 2012. ENVI Documentation, ENVI v5.0.

Jin, X., 2012. ENVI Image Registration. Exelis Visual Information Solutions Whitepaper, $26 \mathrm{p}$. 